\title{
Kolorektales Karzinom als Notfall
}

Patienten mit Darmkrebs, die sich bei Erstdiagnose als Notfälle präsentieren, haben zuvor oft untypische Symptome. Aber nicht immer: Ein erheblicher Prozentsatz könnte laut Studienergebnissen früher erkannt werden.

Wie Daten aus der internationalen Literatur belegen, werden zwischen $14 \%$ und 33\% der kolorektalen Karzinome notfallmedizinisch diagnostiziert. Die betroffenen Patienten werden seltener in kurativer Absicht behandelt, ihre Überlebenschancen sind geringer.

Die Epidemiologin Cristina Renzi vom University College London wollte wissen, ob die Notfallpatienten mit Darmkrebs im Jahr vor der Diagnose einen Hausarzt aufgesucht hatten, welche Symptome und Beschwerden sie gehabt hatten und wie sich das Spektrum von jenem unterschieden hatte, das nicht notfallmäßig diagnostizierte Darmkrebsfälle aufweisen.

\section{Über 95\% aller Notfallpatienten waren zuvor beim Hausarzt}

Zusammen mit Kollegen verknüpfte Renzi dafür die Daten von Patienten mit Darmkrebserkrankungen in England aus den Jahren 2005 und 2006 mit einer Forschungsdatenbank zur hausärztlichen Versorgung. Ihre Ergebnisse haben die Wissenschaftler im British Journal of Cancer veröffentlicht.

\section{Bei Warnsymptomen sollte der} Hausarzt an Darmkrebs denken.
In die Analyse flossen die Angaben zu 1606 Patienten ein, 1029 mit Kolon- und 577 mit Rektumkarzinom. 35\% der Dickdarm- und 15\% der Rektummalignome waren im Zuge einer Notfallversorgung diagnostiziert worden. Im Jahr vor der Diagnose - die drei Monate unmittelbar davor ausgeschlossen, weil Symptome während dieses Zeitraums womöglich bereits mit der Notfallsituation zusammenhängen - waren mehr als $95 \%$ der Notfallpatienten bei ihrem Hausarzt gewesen, wie übrigens auch die Patienten mit regulär diagnostiziertem Krebs.

\section{Relevante Symptome wurden übersehen}

Für Darmkrebs typische, spezifische wie unspezifische Symptome und Beschwerden waren bei den späteren Notfallpatienten seltener festzustellen gewesen (Kolonkrebs: $48 \%$ vs. $71 \%$ bei den regulär Diagnostizierten; Rektumkrebs: $49 \%$ vs. 61\%). Auch Alarmsymptome wie Anämie, rektale Blutung oder veränderte Darmgewohnheiten traten bei Notfallpatienten im Jahr vor der Diagnose weniger häufig auf.

Doch immerhin $18 \%$ der Notfallpatienten mit Kolonkrebs und 23\% der Notfallpatienten mit Rektumkarzinom waren mit solchen Warnzeichen beim Hausarzt vorstellig geworden. $16 \%$ waren dreimal oder öfter mit relevanten Symptomen zum Arzt gegangen.

Renzi und Mitarbeiter betonen, das Hintergrundmuster der Arztbesuche der späteren Notfallpatienten unterscheide sich nicht von jenem der Darmkrebspatienten, die regulär diagnostiziert würden.

Im Jahr vor der Diagnose nehme die Zahl der Arztbesuche aber markant zu. „Obwohl die Notfalltumoren seltener mit typischen Symptomen einherzugehen scheinen“, so die Forscher, „besteht womöglich bei einem Fünftel von ihnen die Gelegenheit für eine frühere Diagnose.“

(Robert Bublak)

Renzi Cet al. Brit J Cancer 2016; 115: 866-875

\section{Helfen Antibiotika bei Divertikulitis?}

Niederländische Forscher haben $528 \mathrm{~Pa}$ tienten mit akuter unkomplizierter Divertikulitis untersucht. Die mediane Dauer bis zum Abklingen der Divertikulitis unterschied sich zwischen Patienten, die antibiotisch behandelt wurden und solchen, die lediglich überwacht wurden, nicht signifikant (14 vs. 12 Tage). Auch mit Blick auf Rezidive (3,4\% vs. $3,0 \%)$ und Mortalität $(1,1 \%$ vs. $0,4 \%)$ waren keine Differenzen festzustellen.

Daniels L et al. Br J Surg 2016, online 30. September; doi: 10.1002/bjs.10309

\section{Methotrexat wirkt bei Colitis ulcerosa}

Im Rahmen der randomisierten placebokontrollierten METEOR-Studie wurden Sicherheit und Wirksamkeit von parenteralem Methotrexat (MTX) bei 111 Patienten mit Colitis ulcerosa untersucht. Nach 16 Wochen hatten $42 \%$ der mit MTX behandelten Probanden eine steroidfreie klinische Remission erreicht. In der Placebogruppe waren es $24 \%$. Der Unterschied war statistisch signifikant.

Carbonnel Fet al. Gastroenterology. 2016;

150(2):380-8

\section{Endoskopie senkt Sterberate}

In einer Untersuchung zu knapp 30.000 Barrett-Patienten fanden Forscher aus Houston Hinweise, wonach bei Ösophaguskarzinompatienten, deren Tumor bei einer Kontrolluntersuchung entdeckt wird, die krebsbedingte Sterblichkeit halb so hoch $(53 \%)$ ist wie bei Betroffenen, deren Tumor nicht bei einer solchen Kontrolle gefunden wird. Nach diesen Daten scheint eine regelmäßige Kontrolle zu einer früheren Tumordiagnose und einer besseren Prognose zu führen.

El-Serag HB et al. Gut 2016;65:1252-1260 